\title{
Accuracy evaluation of 2D geometric correction models: Iraq as a case study
}

\author{
Abdul Razzak Ziboon ${ }^{1, *}$, Israa Mohammed $^{1}$ \\ ${ }^{1}$ Building and Construction Engineering Department, University of Technology, Baghdad, Iraq
}

\begin{abstract}
One of the most important preprocessing methods for remote sensing data and geometrical distrortion is the geometric correction. In this research, several mathematical models were used in two dimensions case and were compared to each other to determine the accuracy of each mathematical model when used in three different regions with different terrains. Three high-resolution satellite images of the QuickBird satellite (a flat area, a hilly area and a mountain area) of Iraq have been used in this work. The flat area is chosen in Baghdad, while the hilly and mountain areas are chosen in Irbil in the north of Iraq. In this research, the mathematical models used are the first and second order polynomials, as well as the projective transform. All of these models were applied to all different topographic areas and their accuracy was assessed based on the Matlab program. The results of the models in the three areas studied indicate that the best precision is achieved with the second order polynomial model, while the worst precision is obtained with the first order polynomial model. On the other hand, the precision of the projective transformation is almost similar to the precision of the first order polynomial.
\end{abstract}

\section{Introduction}

Remote sensing data is collected using several techniques and extensions. For all these systems there are errors associated with the process of data collection. Therefore, preprocessing of the remote sensing data must be done prior to the beginning of the analysis process to eliminate these errors.

The process of image restoration is usually applied to remove distortion, deformation and reduction of noise that are associated with image processing. The image restoration process produces a corrected image that matches as far as possible with the original scene from geometrical and radiological perspective [1].

To correct remote sensing information, internal and external distortions should be considered. Internal distortions are errors that occur within the sensor. These are usually considered as systematic errors and can usually be removed using a mathematical calibration model.

External distortions occur as a result of deviation of the sensing system, changes in the atmosphere and terrain of the earth. These errors are nonsystematic errors and can be eliminated by using control points that appear in the imaged scenes [2].

\section{Geometric correction models}

Geometric correction models are usually simple and do not need to know the components of the sensor physics.
In this sense, these mathematical models must have the ability to link the image coordinates with the ground coordinates [3].

In this paper, several two dimensional models were used for transformation combined with a number of ground control points. Thesle models are usually available in many image processing programs.

The mathematical models used in this paper are:

- 2D Polynomial Model.

- Projective Transformation Model.

\section{1 (2D) Polynomial Model}

Polynomial models are usually used to transform between two different coordinate systems. The transformation order depends mainly on the distortion of the image, the number of control points and the terrain. Usually corrections are only first order or second order. Unwanted results from high-order corrections are the result of the use of complex transformation equations. For the purpose of fitting all ground control points there will be significant distortion in the transformed image. Because the two-dimensional polynomial model does not take into consideration the elevations of ground control points, these mathematical models are more suitable for the flat imaged areas, ie when the image is not affected by terrain. It is usually used in images that have few or small distortions [4]. The following functions represent the general form of the two-dimensional polynomial transformation:

- 1st order polynomial.

"Corresponding author: razzak1956@yahoo.com 


$$
\begin{gathered}
x=a+b X+b Y \\
y=d+e X+f Y
\end{gathered}
$$

$-2^{\text {nd }}$ order polynomial.

$$
\begin{aligned}
& x=a+b X+c Y+d X Y+e X^{2}+f Y^{2} \\
& y=g+h X+i Y+j X Y+k X^{2}+l Y^{2}
\end{aligned}
$$

Where $(\mathrm{x}, \mathrm{y})$ represents the image coordinates of the ground control points of the original input image, while $(\mathrm{X}, \mathrm{Y})$ represents the ground coordinates of the ground control points that are observed using GPS and ( $a, b, c$ to 1) represent the transformation parameters [5].

\subsection{Projective transformation Model}

$$
\begin{aligned}
& x=(a+b X+c Y) /(g X+h Y+1) \\
& y=(d+e X+f Y) /(g X+h Y+1)
\end{aligned}
$$

The above equations are called projective transformation equations. To every point $\mathrm{x}, \mathrm{y}$ in one plane that represented by image coordinates of the ground control points of the original input image there corresponds a point $(\mathrm{X}, \mathrm{Y})$ in the other plane represented by the ground coordinates of the ground control points that are observed using GPS, provided that the common denominator is not zero [6]. If the denominator becomes zero then the corresponding point moves to infinity. It is called vanishing point. The ( $a, b, c$ to $h$ ) are the eight transformation parameters [7].

\section{Study areas}

\subsection{Study area (1)}

The first study area is chosen in Baghdad city in the middle of Iraq. The area has an elevation range of between $(32-47 \mathrm{~m})$, and can be considered as a flat area. Ground control points and check points were distributed as shown in Figures (1) and (2) respectively.

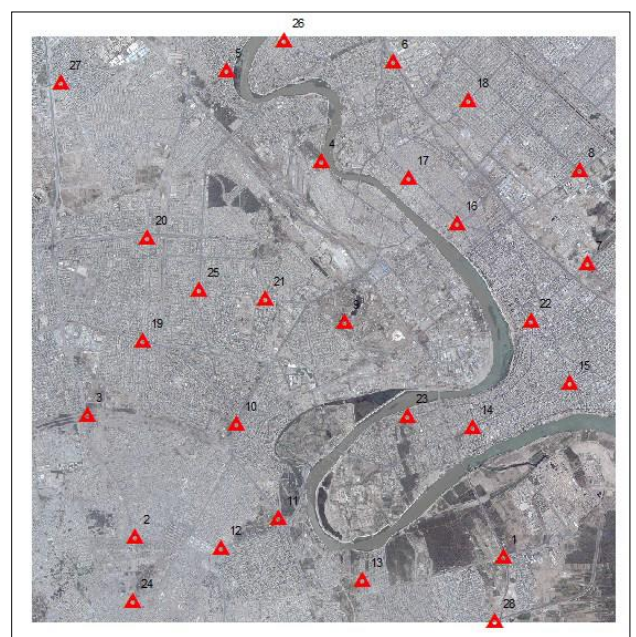

Fig. 1. The first study area with GCPs distribution

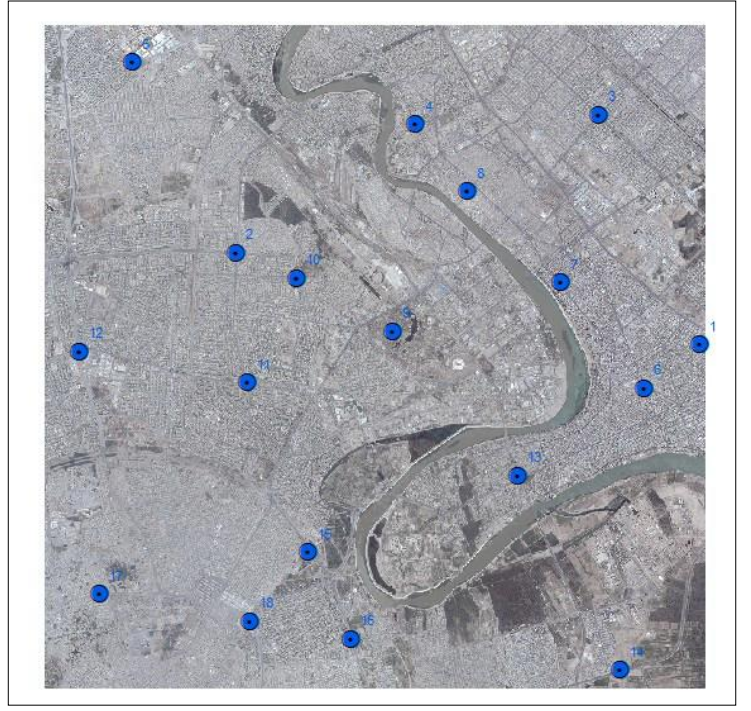

Fig. 2. The first study area with CPs distribution

\subsection{Study area (2)}

The second study area is chosen in the center of Irbil city at the north of Iraq. This area has an elevation range of between $(377-528 \mathrm{~m})$, and can be considered as hilly area. Ground control points and check points were distributed as shown in Figures (3) and (4) respectively.

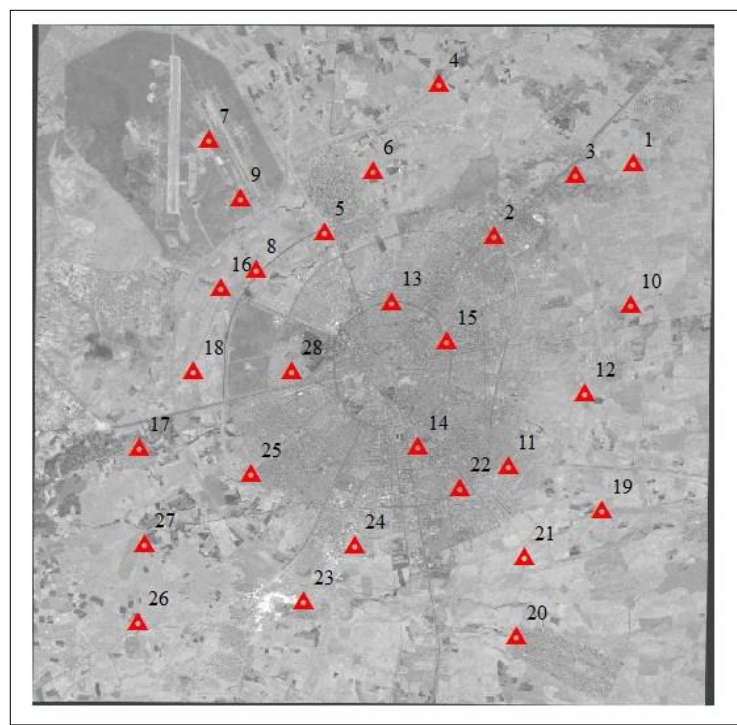

Fig. 3. The second study area with GCPs distribution 


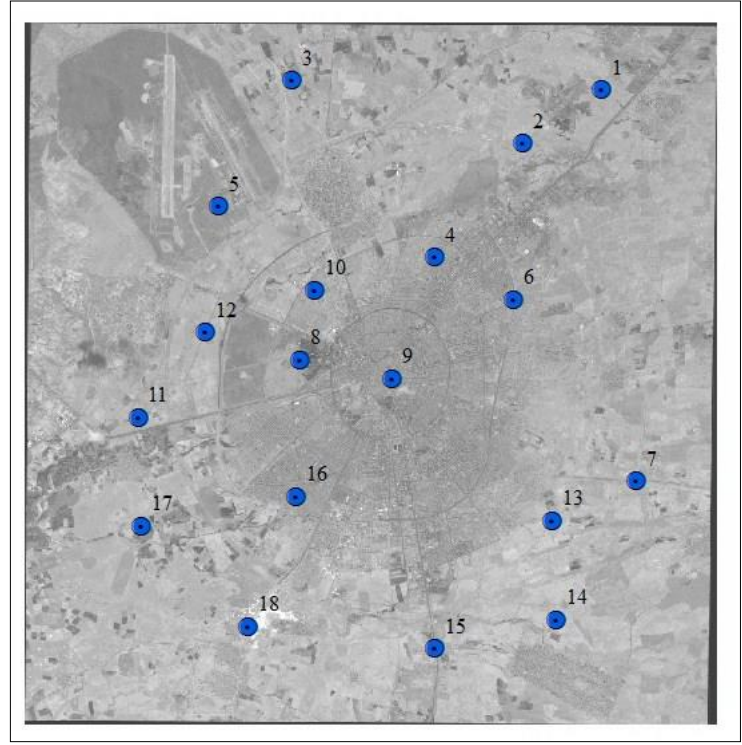

Fig. 4. The second study area with CPs distribution

\subsection{Study area (3)}

The third study area is chosen in Soran, Irbil city at the north of Iraq. The area has an elevation range of between (1450-3150 m), and can be considered as mountain area. Ground control points and check points were distributed as shown in Figures (5) and (6) respectively.

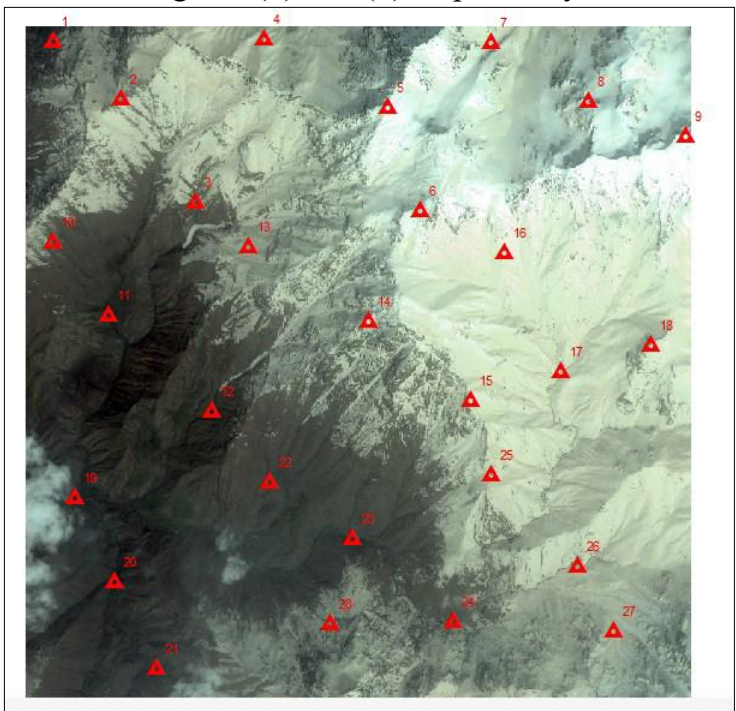

Fig. 5. The third study area with GCPs distribution

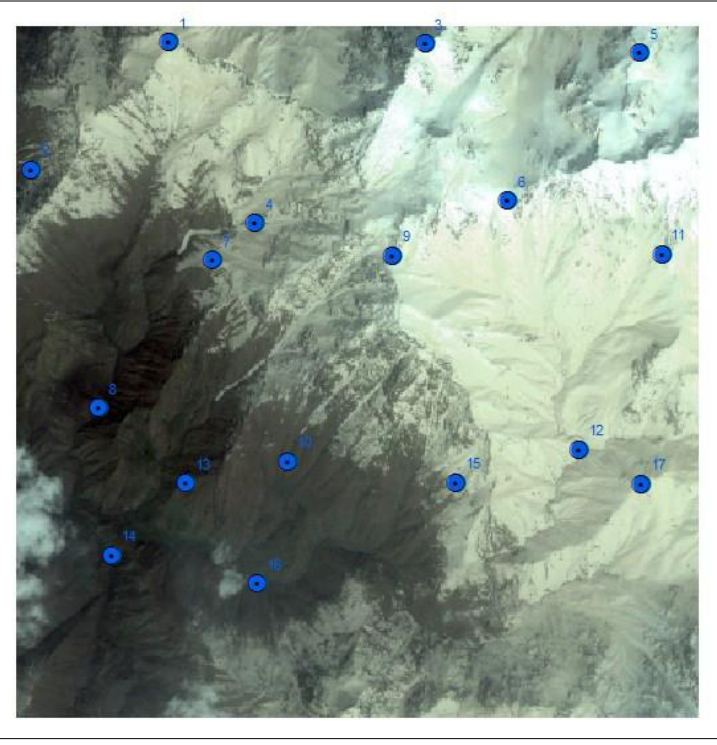

Fig. 6. The third study area with CPs distribution

\section{Results and discussion}

In this paper, different geometric correction mathematical models are applied using three high resolution satellite QuickBird images (panchromatic 0.6 $\mathrm{m}$ in spatial resolution) for three different terrain areas (flat area, hilly area and mountain area) in Iraq. All the geometric models which are used, utilized the ground control points GCPs in order to determine the transformation parameters between the image and the ground. A total number of (46) GCPs were selected, well distributed over each of the three study areas, (28) points were used as control points and the rest of them were considered as check points. The ground coordinates of all GCPs were collected through the DGPS, type (Leica GPS SR20) with the accuracy of $10 \mathrm{~mm}+2 \mathrm{ppm}$ (Rapid Static). The selection of GCPs targets is as accurate as possible. The (TRMSE) for GCPs and check points have been calculated for all models in order to find the best model. All these models are evaluated through the Matlab environment facilities [8].

The geodetic parameters used in DGPS coordinates characteristics and the images information are given in Table 1, this information was used in all methods.

Table 1. The used geodetic parameters and images information

\begin{tabular}{|c|c|c|c|}
\hline Parameter & $\begin{array}{c}\text { QuickBird } \\
\text { (1) }\end{array}$ & $\begin{array}{c}\text { QuickBird } \\
\text { (2) }\end{array}$ & $\begin{array}{c}\text { QuickBird } \\
\text { (3) }\end{array}$ \\
\hline Image type & panchromatic & panchromatic & panchromatic \\
\hline Spatial Resolution & $0.6 \mathrm{~m}$ & $0.6 \mathrm{~m}$ & $0.6 \mathrm{~m}$ \\
\hline Map Projection & UTM & UTM & UTM \\
\hline Datum & WGS 84 & WGS 84 & WGS 84 \\
\hline Zone Number & 38 & 38 & 38 \\
\hline Acquisition Date & 2008 & 2008 & 2008 \\
\hline $\begin{array}{c}\text { Measurement } \\
\text { Method }\end{array}$ & DGPS & DGPS & DGPS \\
\hline
\end{tabular}


The summary of the results and TRMSE conclusion using the three models for the three study areas can be illustrated in Table 2 .

The results of this table show that the 2nd Order 2D Polynomial model is the best model because the best accuracy is achieved with this model. This is due to the nonlinear curve fitting of this model for GCPs distribution. These results ensure that the higher the order of the function, the better is the fit to the GCPs. Also transformation of the 2 nd order is nonlinear transformation that can be used to fit the exact location of point [8].

On the other hand, the worst accuracy is obtained with 1 st order 2D polynomial model and the accuracy of projective transformation model is nearly similar to the accuracy of 1 st order 2D polynomial model.

Table 2. The summary of results for the three study areas

\begin{tabular}{|c|c|c|c|c|c|c|}
\hline \multirow[t]{2}{*}{ Model } & \multicolumn{2}{|c|}{$\begin{array}{l}\text { TRMSE } \\
\text { (pixel) Flat } \\
\text { Area }\end{array}$} & \multicolumn{2}{|c|}{$\begin{array}{c}\text { TRMSE } \\
\text { (pixel) Hilly } \\
\text { Area }\end{array}$} & \multicolumn{2}{|c|}{$\begin{array}{c}\text { TRMSE } \\
\text { (pixel) } \\
\text { Mountain } \\
\text { Area }\end{array}$} \\
\hline & Cont & Chck & Cont & Chck & Cont & Chck \\
\hline $\begin{array}{r}\text { 1st Order 2D } \\
\text { Polynomial }\end{array}$ & 0.6042 & 0.6494 & 0.6914 & 0.7528 & 0.8003 & 0.8508 \\
\hline $\begin{array}{l}2^{\text {nd }} \text { Order 2D } \\
\text { Polynomial }\end{array}$ & 0.5433 & 0.6153 & 0.6436 & 0.7025 & 0.7113 & 0.7875 \\
\hline $\begin{array}{c}\text { Projective } \\
\text { Transformation }\end{array}$ & 0.5878 & 0.6337 & 0.6787 & 0.7206 & 0.7867 & 0.8467 \\
\hline
\end{tabular}

\section{Conclusions}

- The results of 2D models in the three studied areas indicate that the best accuracy is obtained with the second order polynomial model, while the worst accuracy is obtained with the $1^{\text {st }}$ order polynomial model. On the other hand, the accuracy of projective transformation is nearly similar to the accuracy of $1^{\text {st }}$ order polynomial. Therefore, in case of using 2D models, the $2^{\text {nd }}$ order polynomial is recommended to achieve high geometric accuracy.

- Due to the best fitting of nonlinear curve to GCPs distribution, the precision of second order polynomial transformation model is better than that of first order polynomial transformation model.

- In the results of $2 \mathrm{D}$ polynomial and projection transformation models, a linear increase of TRMSE is found, passing from a flat area to more rugged terrains, reaching a maximum value of about ( 0.8 pixel) with a polynomial of first order in the mountain area. Therefore, the 2D models are recommended only in case of flat areas, but they failed in the case of more rugged terrains.

- In the process of calculating the coefficients of mathematical models by least square adjustment solution, the results of Gauss elimination method is more accurate than the results of inverse method especially in case of complex models such as $2^{\text {nd }}$ order 2D polynomial. This is due to the more complicated equation and huge matrices of these models that are required in calculating these coefficients.

\section{References}

1. John R. J. Introductory Digital Image Processing (Pearson, 2016)

2. T Toutin. IEEE, 40, 2241, (2002).

3. Ali O. Accuracy Assessment of the DEM and Orthoimage Generated from ASTER (METU, 2005),

4. C. V. Tao,Y. Hu, PE\&RS, 67, 1347, (2001).

5. M. Hosseini J. Amini, ISPRS H. W., (2005).

6. F. Samadzadegan, A. Milanlak, M. Majdabadi In XXXVI ISPRS (2005).

7. Edward M. M., James S. B. Introduction to Modern Photogrammetry (Wiley, 2001).

8. Ali, B. H. Enhancing The Accuracy of Geometric Correction of High Resolution Satellite Images (UoB, 2006). 